\title{
Construção e Avaliação de uma Intervenção de Planejamento de Carreira para Estudantes Universitários
}

\author{
Cássia Ferrazza Alves' \\ Marco Antônio P. Teixeira' \\ ${ }^{1}$ Universidade Federal do Rio Grande do Sul, Rio Grande do Sul, Porto Alegre, Brasil
}

\begin{abstract}
Resumo
Este estudo tem por objetivo apresentar o desenvolvimento e a avaliação de uma intervenção em grupo na área de planejamento de carreira voltada a estudantes universitários, com base no modelo teórico Hope-Action Theory. Participaram 35 estudantes (22 do grupo de intervenção e 13 do grupo de comparação) com idades entre 19 e 48 anos $(M=23,46 ; D P=5,38)$, de ambos os sexos. O grupo experimental apresentou mudanças mais robustas em termos de tamanho de efeito nas variáveis que avaliavam uma orientação positiva para o futuro (esperança e otimismo) e aspectos relativos à adaptabilidade de carreira. Discute-se acerca de intervenções voltadas para o público universitário e seus desafios, considerando a interface com a saúde mental, além da importância de intervenções pautadas em modelos teóricos consistentes e com instrumentos que possam avaliá-las. Palavras-chave: estudantes universitários, esperança, orientação vocacional, desenvolvimento profissional
\end{abstract}

Construction and Evaluation of a Career Planning Intervention for College Students

\begin{abstract}
This study aims to present the development and evaluation of a group intervention in the area of career planning aimed at university students, based on the Hope-Action Theory model. A total of 35 students (22 in the intervention group and 13 in the comparison group) participated, aged between 19 and 48 years $(M=23.46$; $\mathrm{SD}=5.38)$, both sexes. The experimental group presented more robust changes in terms of effect size in the variables that evaluated a positive orientation to the future (hope and optimism) and aspects related to career adaptability. We discussed the importance of interventions aimed at undergraduate students and their challenges, considering the interface with mental health, besides the importance of interventions based on consistent theoretical models and with instruments that are able to evaluate them.

Keywords: college students, hope, vocational guidance, professional development.
\end{abstract}

Construcción y Evaluación de una Intervención de Planificación de Carrera para Estudiantes Universitarios

\begin{abstract}
Resumen
Este estudio tiene por objetivo presentar la construcción y evaluación de una intervención en equipos en el área de planeamiento de carrera con estudiantes universitarios, basada en el modelo teórico Hope-Action Theory. Participaron 35 estudiantes (22 del grupo intervención y 13 del grupo de comparación) con edades de 19 a 48 años $(M=23,46 ; D P=5,38)$, de ambos sexos. El grupo experimental presentó cambios más robustos en términos del tamaño del efecto de las variables que evaluaban una orientación positiva para el futuro (esperanza y optimismo) y aspectos relativos en la adaptabilidad de carrera. Se discute acerca de la importancia de intervenciones dirigidas al público universitario y sus desafíos, considerando la interfaz con la salud mental, así como la importancia de las intervenciones basadas en modelos teóricos consistentes e instrumentos que puedan evaluarlas. Palabras clave: estudiantes universitarios, esperanza, orientación vocacional, desarrollo profesional.
\end{abstract}

Atualmente, o mercado de trabalho tem sido influenciado por um contexto social e econômico considerado cada vez mais volátil, incerto, complexo e ambíguo (conhecido como VUCA). Verifica-se que as perspectivas profissionais atualmente são menos definidas e previsíveis, com transições mais frequentes. Por isso, identifica-se a importância da construção de metas profissionais coerentes com as trajetórias de vida dos indivíduos. Ao mesmo tempo em que é importante estabelecer metas, o mundo contemporâneo exige dos indivíduos o desenvolvimento de habilidades e competências para lidar com as transições e aprimorar o gerenciamento de suas carreiras. Logo, torna-se relevante que o indivíduo antecipe mudanças, desenvolva percepção de controle e responsabilidade pelas escolhas de carreira (Savickas et al., 2009).

Considerando essas mudanças contextuais, que interferem inevitavelmente na carreira dos indivíduos, torna-se relevante desenvolver intervenções a fim de auxiliar na elaboração de metas e, consequentemente, de um planejamento de carreira. O modelo teórico Hope-Action Theory (HAT; Niles, Amundson, \& Neault, 2010a; Niles, Amundson, \& Yoon, 2019) tem sido utilizado para subsidiar intervenções voltadas para desenvolvimento de carreira dos indivíduos, com foco na construção de metas e planos profissionais (Niles, 
Yoon, Balin, \& Amundson, 2010b; Niles, In, \& Amundson, 2014), o qual possui um instrumento utilizado para avaliar a intervenção, denominado Hope-Action Inventory (HAI; Niles et al., 2010b).

Esse modelo considera a esperança como um aspecto central, sendo composto por atitudes e comportamentos necessários para desenvolver e/ou sustentar a esperança (Niles et al., 2019). Por isso, essa teoria integra três grandes teorias: a de Esperança (Snyder, 2002), a da Agência Humana (Bandura, 2001) e a da Carreira Proteana (Hall, 1996) a fim de promover um modelo teórico que pudesse ser efetivo no gerenciamento de carreira dos indivíduos (Niles et al., 2019). Além disso, o HAT adota uma série de competências, denominadas de "bope-action competencies", que incluem as etapas do modelo, a saber: (a) esperança; (b) reflexão de si, (c) clareza de si, (d) visionamento, (e) estabelecimento de metas e planejamento, (f) implementação e adaptação.

A esperança, primeira etapa do modelo, é compreendida como um estado emocional positivo que emerge da interação entre o estabelecimento de metas, rotas e agência. As metas são os objetivos delineados pelo indivíduo e as rotas, ou caminhos, são as formas para atingir as metas (Snyder, 2002). A agência envolve a habilidade para fazer escolhas e delinear planos de ação de forma intencional além de dar forma apropriada a cursos de ação e de motivar e regular a sua execução (Bandura, 2001).

A reflexão de si, segunda etapa do modelo, propõe o questionamento sobre os interesses, habilidades e valores além de identificar as experiências que contribuíram para o indivíduo ser quem ele é no momento da intervenção (Niles et al., 2010b; Niles et al., 2019). Com essa reflexão, é possível desenvolver a clareza de si, terceira etapa do modelo que se refere à construção de sentido das experiências vividas pelo indivíduo, além de seus interesses e habilidades, que o auxiliam no direcionamento de sua carreira (Niles et al., 2010b).

Por meio da clareza de si, é possível selecionar as opções que melhor respondem aos interesses do indivíduo (Niles et al., 2019) e, com isso, explorar as possibilidades desejadas por meio do visionamento, quarta etapa do modelo, em que se procura visualizar o maior número de opções possíveis para, em seguida, priorizar algumas dessas possibilidades. Após, é importante selecionar as metas desejadas, sendo esta a quinta etapa do modelo - estabelecimento de metas e planejamento. Ao estabelecer as metas, são delineadas também as rotas, isto é, o planejamento para atingir as metas (Niles et al., 2010b; Niles et al., 2019). Com isso, o indivíduo executa seu planejamento, sendo essa a última etapa do modelo - implementação e adaptação. Ao colocar em prática o planejamento, é possível deparar-se com obstáculos ou até mesmo com novas informações, que podem qualificá-lo ou modificar as metas do indivíduo, sendo necessária a disposição do indivíduo para adaptar seus planos e/ou metas (Niles et al., 2014).

Embora o modelo seja dividido em etapas sistematizadas com certa linearidade que organizam o processo do aconselhamento de carreira (e o próprio processo de planejar a carreira), é importante elucidar que, em alguns momentos, é provável que etapas iniciais sejam novamente resgatadas ao final do processo ou, até mesmo, durante o processo. A interação entre a pessoa e o contexto requer disponibilidade do indivíduo para refletir sobre si, contribuindo para a clareza de autoconceito (clareza de si) e para seus objetivos profissionais, respondendo de forma flexível e adaptativa às mudanças em si e no mercado de trabalho, aspecto importante na adaptação dos planos, por exemplo (Niles et al., 2019).

Desse modo, o modelo HAT está próximo conceitualmente à adaptabilidade de carreira, variável importante no desenvolvimento de carreira dos indivíduos, uma vez que ambos os conceitos envolvem orientação para o futuro (Ginevra, Pallini, Vecchio, Nota, \& Soresi, 2016). A esperança permite que o indivíduo possa adaptar seus planos diante dos obstáculos ou aspectos não planejados (Niles et al., 2019) e a adaptabilidade de carreira está associada aos recursos que o indivíduo dispõe em lidar os desafios de carreira, sendo necessária a preocupação do indivíduo ante seu futuro profissional (Savickas, 1997).

Tendo em vista que o contexto universitário é um ambiente em que os estudantes estão próximos a diferentes oportunidades de desenvolvimento profissional, verifica-se um espaço propício para o desenvolvimento de intervenções de carreira a fim de auxiliar os estudantes a refletir sobre seu percurso profissional (Lima-Dias \& Soares, 2012). Ao investigar na literatura intervenções voltadas para o planejamento de carreira de estudantes universitários (Obi, 2015; Park, Rie, Kim, \& Park, 2018; Spurk, Kauffeld, Barthauer, \& Heinemann, 2015; Talib, Salleh, Amat, Ghavifekr, \& Ariff, 2015) ou de pós-graduação (Koen, Klehe, \& Vianen, 2012), evidenciam-se resultados positivos, sendo esses programas desenvolvidos em grupo (Koen et al., 2012; Park et al.,2018), de forma individual (Obi, 2015; Spurk et al., 2015), ou ao longo de uma disciplina da graduação (Talib et al., 2015).

Psico-USF, Bragança Paulista, v. 25, n. 4, p. 697-709, out./ der. 2020 
Quanto ao período de tempo do desenvolvimento das intervenções, estes foram variáveis, desenvolvidos em forma de workshop (um encontro) (Koen et al., 2012) ou com mais encontros (entre três e nove) (Obi, 2015; Park et al., 2018; Spurk et al., 2015; Talib et al., 2015). Ainda que as propostas desenvolvidas por esses autores tivessem suas singularidades com base em diferentes abordagens teóricas (por exemplo, no paradigma Life-design ou na teoria de John Holland) (Koen et al., 2012; Obi, 2015; Talib et al., 2015), estas foram semelhantes. As temáticas dos encontros versaram sobre autoconhecimento, conhecimento do mercado de trabalho e delineamento de planos de carreira (Koen et al., 2012; Park et al., 2018; Spurk et al., 2015; Talib et al., 2015); ou somente a delimitação de metas profissionais (Obi, 2015).

Quanto aos resultados, os participantes do grupo experimental apresentaram incrementos nos aspectos da adaptabilidade de carreira (Koen et al., 2012), de variáveis relacionadas a clareza de metas e planos de carreira (Obi, 2015; Talib et al., 2015), incremento na percepção de otimismo e de sucesso na carreira (Spurk et al., 2015) e na autoeficácia para tomada de decisões profissionais (Park et al., 2018). Tendo em vista que a esperança é um construto de orientação ao futuro e sua definição engloba a construção de metas e de rotas, verifica-se que, embora essas intervenções não utilizaram o construto da esperança, a orientação para metas e planos profissionais esteve presente.

Ao investigar pesquisas sobre intervenções que visam promover a esperança em estudantes universitários, são encontrados programas de curta duração (tipicamente workshops de encontro único com noventa minutos de duração, em média) (Davidson, Feldman, \& Margalit, 2012; Feldman \& Dreher, 2012; Feldman \& Kubota, 2015). De um modo geral, consistem em uma explicação dos aspectos que compõe o conceito (metas, rotas e agência), seguida da execução de uma tarefa na qual os participantes devem elencar uma meta (não necessariamente de cunho profissional) com três rotas, um obstáculo para cada rota e uma maneira de contorná-lo (Davidson et al., 2012; Feldman \& Kubota, 2015). Embora os resultados desses estudos mostrem efeitos positivos com incremento da esperança após a intervenção, observa-se que esses resultados não são estáveis no follow up para todos os indivíduos (Davidson et al., 2012; Feldman \& Kubota, 2015). Esses resultados podem estar relacionados ao fato das intervenções seguirem modelo de encontro único, sendo relevante a construção de programas com maior tempo de duração (Feldman \& Kubota, 2015).

Em contrapartida, as intervenções construídas a partir do modelo HAT, as quais abordam os aspectos que compõem o modelo teórico (com técnicas), são realizadas com refugiados (Yoon, Bailey, Amundson, \& Niles, 2019), desempregados (Amundson, Goddard, Yoon, \& Niles, 2018) e trabalhadores (Clarke, Amdunson, Niles, \& Yoon, 2018), apresentando resultados positivos após a intervenção, avaliados pelo HAI. Em termos de duração e configuração, elas foram variáveis, sendo evidenciadas intervenções desenvolvidas em dez encontros (parte em grupo e individual; Yoon, 2019); estudo de comparação entre encontros presenciais individual (dois encontros) e uma intervenção on-line (programa de duas semanas; Amundson et al., 2018), além de um programa desenvolvido em um encontro único (Clarke et al., 2018).

Embora essas intervenções tenham apresentado resultados positivos, com incremento dos resultados do HAI, verifica-se que são intervenções com diferentes configurações e direcionadas para públicos diversos. Apesar disso, esse modelo teórico diferencia-se de outros modelos de intervenção na área de carreira por abordar, de uma forma sequencial e estruturada, processos relevantes envolvidos nas decisões de carreira. Essa estrutura permite que sejam avaliados aspectos que requerem maior atenção do orientador, ao mesmo tempo em que é possível organizar a própria intervenção em uma sequência que tem relação com o progresso das decisões vocacionais do indivíduo.

O objetivo deste estudo foi, então, desenvolver e avaliar uma intervenção em grupo com estudantes universitários baseada no modelo do HAT (Niles et al., 2010a). A escolha desse modelo teórico como base da intervenção deve-se a sua clareza conceitual e organização sequencial estruturada, o que facilita a compreensão dos processos envolvidos nas decisões vocacionais. Embora existam evidências de que as intervenções individuais tendem a ser mais efetivas do que as grupais (Whiston, Li, Mitts, \& Wright, 2017) é relevante desenvolver intervenções para grupos a fim de abarcar um maior número de pessoas especialmente em contextos educacionais como o do ensino superior, bem como avaliar essas intervenções com instrumentos congruentes ao objetivo dela. A avaliação de intervenções em orientação profissional e aconselhamento de carreira tornam-se imprescindíveis, a fim de destacar para a importância do investimento público ou privado em serviços que proporcionem tais projetos, sendo, nesse 
caso, o contexto universitário (Ambiel, Barros, Pereira, Tofoli, \& Bacon, 2017).

Como hipótese, estima-se que os participantes da intervenção apresentarão ganhos nas dimensões do HAI e em esperança, otimismo e adaptabilidade de carreira. A inclusão de esperança e otimismo como potenciais indicadores de efetividade da intervenção justifica-se pelo fato de que o modelo destaca o papel da esperança no desenvolvimento de carreira, e o otimismo é um correlato da esperança que tem sido investigado em estudos sobre aconselhamento de carreira (Feldman, Davidson, \& Margali, 2015; Feldman \& Kubota, 2015; Santilli, Marcionetti, Rochat, Rossier, \& Nota, 2017). Já a adaptabilidade de carreira foi incluída por ser uma variável bastante utilizada como indicador de efetividade de intervenções de carreira (e. g., Fouad, Ghosh, Chang, Figueiredo, \& Bachhuber, 2016; Nota, Santilli, \& Soresi, 2016; Santilli, Nota, \& Hartung, 2019).

\section{Método}

\section{Delineamento}

Trata-se de uma pesquisa quase experimental, com a composição de um grupo intervenção (GI) e um grupo de comparação (GC) não equivalente (Creswell, 2010), com avaliação antes e depois da intervenção.

\section{Procedimentos e Considerações Éticas}

A pesquisa foi aprovada pelo Comitê de Ética em Pesquisa do (Informação Retirada pela Revista) (protocolo 45001015.2.0000.5334), conforme resolução 510/16 de pesquisas com seres humanos (Brasil, 2016). Todos os participantes do GI e GC responderam aos questionários após a concordância do Termo de Consentimento Livre e Esclarecido (TCLE).

Inicialmente, foi realizado um estudo piloto em uma Instituição de Ensino Superior (IES) pública, em novembro e dezembro de 2015, a fim de verificar a adequação da intervenção ao tempo e ao público proposto. A partir do estudo piloto, foram realizadas algumas alterações na seleção da amostra para a coleta de dados, sendo delimitada a etapa do curso (a partir do $3^{\circ}$ semestre de ingresso na IES), considerando que os alunos de início do curso ainda estavam adaptando-se ao contexto universitário.

O convite aos participantes para o GI foi realizado por meio das coordenações dos cursos, diretórios acadêmicos, empresas júnior e de serviços institucionais voltados para os universitários. Foram oferecidas dez edições da intervenção, sendo que os dados dos participantes das dez edições foram reunidos para compor o grupo experimental. Cada edição foi oferecida em dia e horário pré-estabelecido, sendo que os interessados se inscreviam previamente para participar da atividade. Os participantes do GI responderam aos instrumentos, de sete a dez dias antes da intervenção (T1) de forma on-line e após a intervenção (T2) de forma presencial (ao final do terceiro encontro).

O GC, por sua vez, foi composto por meio de convite em sala de aula para a participação na pesquisa, após indicação de turmas feitas pelas coordenações de cursos. Inicialmente o plano era compor um grupo de comparação formado por pessoas interessadas na intervenção que estivessem em lista de espera. No entanto, como não houve procura elevada pela intervenção, não foi possível implementar esse procedimento. Assim, optou-se por convidar estudantes em sala de aula para participarem da pesquisa. Aqueles que concordaram em participar responderam aos instrumentos presencialmente em sala de aula (T1) e, após três semanas, receberam um link para responderem o questionário on-line (T2). O tempo de três semanas correspondeu à duração da intervenção.

\section{Participantes}

Uma vez que dificuldades de ordem emocional podem comprometer os resultados das intervenções de carreira (Guadassi, Waser, \& Gati, 2015), foi realizado um rastreamento de sinais e sintomas de transtornos psicológicos (especialmente ansiedade e depressão) antes da participação da intervenção, sendo excluídos da pesquisa (tanto no GI quanto no GC). Assim, no momento em que o estudante tinha interesse em participar do grupo (GI), este fazia uma inscrição prévia e respondia aos instrumentos Self-Reporting Questionnaire e à Escala de Depressão, Ansiedade e Estresse - Versão reduzida (DASS -21) (detalhes sobre os instrumentos estão descritos no tópico Instrumentos). Tanto no GI quanto no GC, indivíduos com escore maior do que sete no SRQ-20 e escore severo nas subescalas depressão (somatório dos pontos entre 11 e 13) e ansiedade (somatório dos pontos entre 08 e 09) na DASS-21 não participaram da intervenção, sendo contatados e indicados para acompanhamento psicológico.

Nesse sentido, 95 estudantes inscreveram-se para participar GI, sendo que 35 foram excluídos da amostra. Dos 60 estudantes que poderiam participar das intervenções, apenas 35 iniciaram o grupo e 22 participaram de todos os encontros. No GC, dos 120 estudantes que aceitaram participar do estudo, 21 
foram excluídos da amostra e apenas 13 responderam aos questionários em T2.

Desse modo, 22 indivíduos compuseram o GI, com idades entre 19 e 48 anos $(M=23,9 ; D P=6,1)$ e 13 indivíduos o GC, com idades entre 19 e 32 anos $(M$ $=22,8 ; D P=4,1)$. Todos os participantes eram estudantes do ensino superior e estavam cursando a partir do terceiro semestre. Informações sobre sexo, curso e renda familiar podem ser visualizadas na Tabela 1.

\section{Descrição da Intervenção}

A intervenção, de caráter grupal, foi estruturada em três encontros com tempo de duração de duas horas cada e realizado uma vez por semana, entre junho de 2016 a maio de 2017, conforme o calendário acadêmico. Com esse número de encontros foi possível organizar a estrutura sequencial do modelo do HAT (Niles et al., 2010a) e a proposta de ser uma intervenção voltada para o planejamento de carreira. Para organizar o processo, foi elaborado um portfólio, entregue a todos os participantes no primeiro encontro, contendo as atividades a serem realizadas tanto no encontro (quando faziam parte da proposta) ou como tarefas de casa.

No primeiro encontro, inicialmente, foi identificada a demanda dos participantes e apresentado o modelo do HAT (Niles et al., 2010a) bem como o conceito de esperança. Buscou-se desenvolver aspectos relativos à reflexão e à clareza de si. Por meio da técnica "linha da vida", foi possível compreender os momentos mais importantes da vida dos participantes e que influenciaram na percepção de si. Outras ferramentas foram utilizadas a fim de identificar os valores de carreira e a avaliação de aspectos satisfatórios, insatisfatórios e aprendizados evidenciados a partir de experiências de carreira vivenciadas pelos participantes (esta última tarefa foi direcionada como tarefa de casa e discutida no encontro seguinte).

No segundo encontro, buscou-se identificar as aspirações dos participantes, baseado na etapa do visionamento do modelo teórico (Niles et al., 2010a), e a relação entre as aspirações e os interesses, os valores e a história de vida dos participantes. $\mathrm{Na}$ atividade denominada "O que desejo para meu futuro?", os participantes respondiam o que esperavam do seu futuro e, em seguida, a perguntas norteadoras que procuravam ajudar os orientandos na organização desses objetivos mais amplos em metas possíveis de serem realizadas. Desse modo, foram delimitadas metas a curto, médio e longo prazo, independente do participante de fato desejar executar essa meta, mas como um exercício sobre como construir metas possíveis de serem executadas a partir dos desejos futuros, sendo investigada a possibilidade de realização das metas como tarefa de casa.

No último encontro, foram selecionadas as metas que os participantes gostariam de executar, baseado na etapa Estabelecimento de metas e planejamento (Niles et al., 2010a), os caminhos e possíveis obstáculos para a execução das metas, bem como

Tabela 1.

Características da Amostra

\begin{tabular}{|c|c|c|}
\hline & Grupo intervenção & Grupo Comparação \\
\hline \multirow[t]{2}{*}{ Sexo } & $68,2 \%$ sexo feminino & $69,2 \%$ sexo feminino \\
\hline & $31,8 \%$ sexo masculino & $30,8 \%$ sexo masculino \\
\hline \multirow[t]{3}{*}{ Curso } & $13,6 \%$ engenharia ambiental & $46,2 \%$ nutrição \\
\hline & $\begin{array}{l}\text { 9,1\% nos seguintes cursos (percentual por } \\
\text { curso): design de produto, enfermagem, } \\
\text { engenharia civil, engenharia física, farmácia }\end{array}$ & $\begin{array}{l}\text { 15,4\% nos seguintes cursos (percentual por } \\
\text { curso): engenharia de energia, engenharia } \\
\text { elétrica, licenciatura em dança }\end{array}$ \\
\hline & $\begin{array}{l}4,5 \% \text { nos seguintes cursos (percentual por } \\
\text { curso): administração, biblioteconomia, } \\
\text { ciências econômicas, engenharia de materiais, } \\
\text { letras, licenciatura em dança, logística, } \\
\text { psicologia e química industrial }\end{array}$ & $7,7 \%$ psicologia \\
\hline \multirow[t]{3}{*}{ Renda familiar } & $40,9 \%$ - renda superior a $\mathrm{R} \$ 7.000,00$ & $23,1 \%$ - renda superior a $R \$ 7.000,00$ \\
\hline & $27,3 \%$ - renda entre $\mathrm{R} \$ 4.001,00$ e $\mathrm{R} \$ 7.000,00$ & $15,4 \%$ - renda entre $\mathrm{R} \$ 6.501,00$ e $\mathrm{R} \$ 7.000,00$ \\
\hline & $31,8 \%$ - renda inferior a $\mathrm{R} \$ 3.500,00$ & $61,5 \%$ - renda inferior a $\mathrm{R} \$ 3.500,00$ \\
\hline
\end{tabular}


foram elaboradas formas de contorná-los (Feldman et al., 2015). Também foi discutido com os participantes para quem era importante atingir as metas (por exemplo, a família) e com quem eles gostariam de compartilhar a conquista dela. Após, foi realizado um resumo dos encontros e investigada a percepção dos universitários sobre o que o grupo contribuiu para sua carreira, respondendo, em seguida, ao questionário Tempo 2 (T2). Os encontros e os temas trabalhados podem ser visualizados na Tabela 2 .

\section{Instrumentos}

Ficha de caracterização dos participantes. Foi utilizado um questionário a fim de obter informações sobre idade, sexo, curso de graduação, semestre no curso.

Hope-Centered Career Inventory (HAI) (Niles et al., 2010a adaptado e validado para o Brasil por Alves, 2018). Avalia o nível de esperança em 28 itens, divididos em sete dimensões: esperança, reflexão de si, clareza de si, visionamento, estabelecimento de metas e planejamento, implementação e adaptação. O instrumento apresentou índices adequados de consistência interna para o contexto brasileiro (escala total $\alpha=0,90$; esperança $\alpha=0,82$; reflexão $\alpha=0,82$; clareza de si $\alpha=$ 0,79 ; visionamento $\alpha=0,75$; estabelecimento de metas e planejamento $\alpha=0,76$; implementação $\alpha=0,77$; adaptação $\alpha=0,75)$.

Adult Dispositional Hope Scale (Snyder et al., 1991, adaptada e validada para o Brasil por Pacico, Bastianello, Zanon, \& Hutz, 2013). Avalia o nível de esperança em uma escala com 12 itens, em que quatro itens avaliam rotas e quatro itens avaliam agenciamento. Possui também quatro itens que são filtro e não são inclú́dos para avaliação do escore do sujeito. A escala apresentou índice adequado de consistência interna em estudos de validação da versão brasileira $(\alpha=0,80)$.

Life Orientation Test-Revised (LOT-R, Scheier, Carver, \& Bridges, 1994, adaptado e validado para o Brasil por Bastianello, Pacico, \& Hutz, 2014). Avalia o nível de otimismo em uma escala unidimensional de dez itens. A versão brasileira da escala apresentou índice adequado de consistência interna $(\alpha=0,80)$.

Escala de Adaptabilidade de Carreira (Savickas \& Porfeli, 2012, adaptado e validado para o Brasil por Audibert \& Teixeira, 2015). Tem por objetivo avaliar a adaptabilidade de carreira. A escala apresenta 24 itens, dividida em quatro dimensões: preocupação, controle, curiosidade e confiança. No contexto brasileiro, a escala

Tabela 2.

Características da Intervenção

Encontro

$1^{\mathrm{o}}$ Encontro

$3^{\circ}$ Encontro

\section{Objetivo}

Desenvolver o autoconhecimento através das etapas Reflexão e clareza de si do modelo HAT

Favorecer o comportamento exploratório por meio da etapa Visionamento do modelo HAT

Realizar o planejamento de carreira por meio das etapas Estabelecimento de metas e planejamento do modelo HAT

\section{Conteúdo trabalhado}

- Destacar os momentos mais importantes da vida do participante (linha de vida);

- Conhecer os valores relacionados à carreira; - Tarefa de casa - relembrar e destacar experiências importantes relacionadas a atividades de trabalho ou estudo (inventário pessoal).

- Articular as informações levantadas sobre a história de vida, interesses e valores com as expectativas quanto ao futuro (atividade $\mathrm{O}$ que desejo para meu futuro?);

- Descrever metas a serem realizadas a curto, a médio e a longo prazo;

- Tarefa de casa: investigar a possibilidade das metas serem realizadas.

- Expor para o grupo as metas e a possibilidade de execução das pesquisadas (atividade Quais são minhas metas?);

- Delinear três rotas para cada meta, um obstáculo para cada rota e também uma forma de contorná-lo (atividade Quais são minhas metas?);

- Avaliação dos encontros. 
total e as subescalas apresentaram índices adequados de consistência interna (escala total $\alpha=0,94$; preocupação $\alpha=0,88$; controle $\alpha=0,83$; curiosidade $\alpha=0,88$; confiança $\alpha=0,89$ ).

Self-Reporting Questionnaire (SRQ 20, desenvolvido pela Organização Mundial de Saúde, OMS, validado para o Brasil por Santos, Araújo, \& Oliveira, 2009). É composto por 20 itens que avaliam sintomas de transtorno mental (insônia, fadiga, irritabilidade, esquecimento, dificuldade de concentração e queixas somáticas). É um instrumento de rastreio para transtorno mental, mas não discrimina tipos de transtornos. A versão brasileira apresentou índice adequado de consistência interna $(\alpha=0,80)$.

Escala de Depressão, Ansiedade e Estresse Versão reduzida (DASS-21) (Lovbond \& Lovbond, 1995, adaptado e validado para o Brasil por Machado, 2013). É composta por 21 itens que avaliam sintomas relacionados à depressão, ansiedade e estresse, e apresentou consistência interna adequada $(\alpha=0,85$ ansiedade; $\alpha=0,89$ estresse; $\alpha=0,91$ depressão) no estudo de Machado (2013). A partir do somatório dos escores para cada subescala, pode ser definido um nível de depressão, ansiedade e estresse em normal, médio, moderado, severo e extremamente severo (Lovbond, 1998).

\section{Análise dos Dados}

Foram realizadas análises descritivas dos dados sociodemográficos e das variáveis-alvo da pesquisa, no software Statistical Package for the Social Sciences (SPSS). Inicialmente, foi verificada se a distribuição da amostra não violava os pressupostos da normalidade, sendo realizado o teste de Kolmolgorov-Smirnov. Pelo fato de não violar o pressuposto da normalidade, foram realizados testes $t$ de Student para identificar as diferenças entre as médias dos participantes dos dois grupos e nos diferentes tempos de coleta de dados (T1 e T2), além de terem sido calculados os tamanhos de efeito entre os grupos GI e GC e intragrupos (ANOVAS 2x2).

\section{Resultados}

Os dados descritivos das variáveis investigadas são apresentados na Tabela 2, comparando as médias do grupo intervenção (GI) e de comparação (GC) em T1. Foram identificadas diferenças entre as médias do GI e GC no primeiro tempo de coleta de dados nas subescalas HAI total $[t(33)=-2,46 ; p=0,019]$, clareza de si $[t(33)=-2,27 ; p=0,03]$, estabelecimento de metas [t(33)
$=-2,73 ; p<0,01]$, e preocupação $[t(33)=-3,26 ; p<$ $0,01]$, tendo o GC apresentado médias estatisticamente superiores ao GI.

De modo geral, ao visualizar a Tabela 2 , é possível identificar que, em T1, o GC apresentou médias superiores em relação ao GI, sendo a diferença estatisticamente significativa nas variáveis HAI total, clareza de si, estabelecimento de metas e preocupação. Em T2, as médias de GI, na maior parte das variáveis, foram superiores ao $\mathrm{GC}$, embora as diferenças não sejam estatisticamente significativas. As médias do GC que foram superiores ao do GI em T2, apresentaram tamanho de efeito pequeno (reflexão e clareza de si, estabelecimento de metas e planejamento, implementação, agência e curiosidade), com exceção da dimensão estabelecimento de metas que apresentou tamanho de efeito moderado (Cohen, 1977).

Nos testes $t$ de Student, identifica-se que o GC não apresentou mudanças significativas nas variáveis investigadas (de T1 para T2), exceto para a variável curiosidade, cujo escore aumentou. O GI, por outro lado, apresentou incrementos estatisticamente significativos nas médias para a maior parte das variáveis, exceto visionamento, implementação, agência, controle e confiança.

De um modo geral, portanto, verifica-se que o GI apresentou mudanças estatisticamente significativas de T1 para T2, observando-se uma mudança mais robusta em termos de tamanho de efeito (e com tamanhos de efeitos de médios a grandes), com exceção da implementação, enquanto o GC não apresentou mudanças estatisticamente significativas (exceto curiosidade). Ou seja, os escores do GI aproximaram-se dos escores do GC em T2, o que sugere em efeito positivo da intervenção.

Observa-se que, no GI, as variáveis HAI total e esperança, rotas e otimismo, adaptabilidade de carreira, preocupação e confiança, com exceção do otimismo e do HAI total, apresentaram escores inferiores em T1 em relação ao GC e, em T2, apresentaram escores superiores comparando-as ao GC. Além disso, verifica-se que foram evidenciados tamanhos de efeito grande nas variáveis HAI total $(d=-1,63)$, esperança $(d=-1,25)$, adaptabilidade de carreira $(d=-1,59)$ e preocupação $(d=-2,84)$.

\section{Discussão}

Este estudo buscou avaliar uma intervenção em grupo com estudantes universitários, baseada no 
Tabela 3.

Médias (M), Desvios Padrão (DP) e Tamanhos de Efeito (d) em T1 e T2

\begin{tabular}{|c|c|c|c|c|c|c|c|}
\hline & \multicolumn{3}{|c|}{ T1 } & \multicolumn{2}{|c|}{ T2 } & \multicolumn{2}{|c|}{$\begin{array}{l}\text { Comparação } \\
\text { intragrupos }\end{array}$} \\
\hline & GI & GC & & GI & GC & GI & GC \\
\hline & $\begin{array}{c}M \\
(D P)\end{array}$ & $M(D P)$ & $d$ & $\begin{array}{c}M \\
(D P)\end{array}$ & $M(D P)$ & $d$ & $D$ \\
\hline HAI Total $^{1}$ & $2,99(0,28)$ & $3,22(0,23)$ & $-0,90 * *$ & $3,32(0,36)$ & $3,27(0,30)$ & $-1,63 * *$ & $-0,30$ \\
\hline Esperança & $3,28(0,49)$ & $3,31(0,49)$ & $-0,04$ & $3,62(0,37)$ & $3,29(0,60)$ & $-1,25^{* *}$ & 0,03 \\
\hline Reflexão de si & $3,17(0,49)$ & $3,31(0,51)$ & $-0,28$ & $3,43(0,60)$ & $3,49(0,45)$ & $-0,75^{* *}$ & $-0,59$ \\
\hline Clareza de si & $2,67(0,42)$ & $3,02(0,47)$ & $-0,79 *$ & $3,06(0,67)$ & $3,08(0,50)$ & $-1,13 * *$ & $-0,19$ \\
\hline Visionamento & $3,07(0,48)$ & $3,42(0,62)$ & $-0,64$ & $3,62(0,37)$ & $3,46(0,62)$ & $-2,05$ & $-0,10$ \\
\hline Estab. Metas & $2,71(0,59)$ & $3,23(0,44)$ & $-1,01 * *$ & $3,10(0,57)$ & $3,21(0,53)$ & $-1,06^{*}$ & 0,06 \\
\hline Implementação & $2,75(0,58)$ & $3,02(0,24)$ & $-0,66$ & $2,86(0,70)$ & $3,06(0,37)$ & $-0,27$ & $-0,21$ \\
\hline Adaptação & $3,27(0,47)$ & $3,23(0,51)$ & 0,08 & $3,54(0,41)$ & $3,33(0,55)$ & $-0,97 * *$ & $-0,30$ \\
\hline $\mathrm{ADHS}^{2}$ & $3,62(0,54)$ & $3,92(0,58)$ & $-0,53$ & $3,88(0,69)$ & $3,84(0,55)$ & $-0,67^{*}$ & 0,22 \\
\hline Rotas & $3,64(0,64)$ & $3,86(0,82)$ & $-0,30$ & $3,95(0,72)$ & $3,70(0,65)$ & $-0,72^{*}$ & 0,34 \\
\hline Agência & $3,61(0,56)$ & $3,98(0,56)$ & $-0,67$ & $3,81(0,75)$ & $3,98(0,60)$ & $-0,80$ & 0,00 \\
\hline Lot-R ${ }^{3}$ & $3,86(0,81)$ & $3,73(0,70)$ & 0,17 & $4,23(0,67)$ & $3,61(0,78)$ & $-0,79 * *$ & $-0,26$ \\
\hline Escala de Adap. de Carreira ${ }^{3}$ & $3,37(0,58)$ & $3,70(0,56)$ & $-0,58$ & $3,96(0,59)$ & $3,77(0,54)$ & $-1,59 * *$ & $-0,20$ \\
\hline Preocupação & $3,03(0,66)$ & $3,78(0,65)$ & $-1,14^{* *}$ & $4,19(0,63)$ & $3,86(0,68)$ & $-2,84 * *$ & $-0,19$ \\
\hline Controle & $3,60(0,86)$ & $3,59(0,74)$ & 0,01 & $3,97(0,75)$ & $3,55(0,62)$ & $-0,73^{*}$ & $-0,09$ \\
\hline Curiosidade & $3,12(0,97)$ & $3,51(0,68)$ & $-0,47$ & $3,68(0,80)$ & $3,77(0,61)$ & $-1,00 *$ & $-0,64 *$ \\
\hline Confiança & $3,71(0,59)$ & $3,90(0,73)$ & $-0,29$ & $4,00(0,60)$ & $3,88(0,75)$ & $-0,77$ & $-0,04$ \\
\hline
\end{tabular}

Nota. ${ }^{*} p \leq 0,05,{ }^{* *} p \leq 0,01 .{ }^{1}$ Hope-Centered Career Inventory; ${ }^{2}$ Adult Dispositional Hope Scale, ${ }^{3}$ Life Orientation Test-Revised.

modelo do HAT (Niles et al., 2010a). Os resultados evidenciaram diferenças estatisticamente significativas para as variáveis investigadas no GI, exceto visionamento, implementação, agência e confiança e tamanhos de efeito moderados e altos para todas as variáveis. A ausência de diferenças estatisticamente significativas nessas variáveis pode estar relacionada ao tempo de duração da intervenção e do enfoque. Embora seja uma intervenção baseada em um modelo centrado na esperança, a estrutura da proposta não teve o foco na execução das metas e na adaptação das rotas, aspectos estes ligados à agência. Possivelmente, intervenções que possam acompanhar a execução do planejamento delineado pelo indivíduo podem auxiliar na confiança dele em atingir as metas estipuladas. Diante de obstáculos, que requerem novos posicionamentos do indivíduo, o orientador profissional pode auxiliar na construção de planos alternativos e na motivação do cliente para superar tais obstáculos ao persistir ou remodelar o planejamento (Niles et al., 2019; Thompson, Her, \& Nitzarim, 2014).

O GC também apresentou pequenas alterações entre o T1 e T2, do ponto de vista do tamanho de efeito, sendo que o GI apresentou tamanhos de efeito relativamente maiores que GC. Na variável curiosidade, a diferença identificada em T1 e T2 no GC pode estar relacionada ao fato de ter sido um pequeno estímulo (ou efeito) para os participantes, que responderam o questionário no primeiro momento, desenvolverem a curiosidade a respeito de si e das oportunidades de carreira.

Ao analisar a interação entre os momentos (T1 e T2) e a relação entre o tipo de intervenção (GI e GC), o GI apresentou mudança mais robusta em termos de tamanho de efeito nas variáveis que avaliavam uma orientação positiva para o futuro (dimensão esperança do HAI e otimismo) e aspectos relativos à preocupação com o futuro e à própria adaptabilidade de carreira. 
Possivelmente, participar da intervenção promoveu, nos participantes, maior preocupação com o futuro e disponibilidade para lidar com novos desafios e transições de carreira, possivelmente, por ser uma proposta com foco no delineamento de metas. Para construir as metas, parece ser necessário preocupar-se previamente com o futuro, aspecto básico para o engajamento nas tarefas relacionadas à carreira (Savickas, 2013).

Além disso, no GI, a adaptabilidade de carreira aumentou juntamente com as variáveis otimismo e esperança. Embora não tenha sido analisada a relação entre elas, o aumento da adaptabilidade de carreira, otimismo e esperança são variáveis que têm sido relacionadas a aspectos relativos à decisão de carreira (Ginevra et al., 2016). Possivelmente, o universitário, ao compreender que existe uma continuidade entre as decisões presentes e as decisões futuras, percebe-se como responsável sobre suas decisões (Savickas, 2013). Com isso, pode desenvolver uma orientação positiva para o futuro e confiança para tomar as decisões de carreira (Ginevra et al., 2016; Santilli et al., 2017).

Além disso, a mudança foi observada também no HAI total, escala construída para avaliar o modelo do HAT (Niles et al., 2010a), evidenciando o potencial dessa intervenção para o público universitário. Sugere-se que, para fins de aplicabilidade dessa intervenção nesse contexto, que sejam realizados novos encontros, posteriores à intervenção, em que o orientador acompanhe a execução dos planos pelos participantes a fim de auxiliá-los nos possíveis obstáculos e desafios encontrados na implementação do planejamento. Quanto às implicações práticas, verifica-se que planejar a carreira está associado a uma maior articulação dos universitários às demandas do mercado de trabalho, considerando que a universidade busca desenvolver no estudante $o$ pensamento crítico-reflexivo e a autonomia para que ele construa seu percurso profissional de acordo com seus interesses. Contudo, ainda carecem de disciplinas, inseridas na matriz curricular, que abordem a construção desse percurso acadêmico, sendo necessária a inclusão de serviços institucionais que possibilitem a realização de atividades voltadas para o aconselhamento de carreira (Ramos et al., 2018).

Ao mesmo tempo, torna-se relevante refletir sobre a baixa procura dos universitários para grupos com foco no planejamento de carreira e o número elevado de estudantes que não puderam participar da intervenção pelo fato de possuírem sintomas característicos de adoecimento mental. Nesse caso, possivelmente pensar a carreira possa ser difícil em virtude de que outras preocupações podem estar afetando esses indivíduos. Contudo, possivelmente realizar um tratamento combinado entre a psicoterapia e o aconselhamento de carreira possa ser uma alternativa para auxiliá-los na construção de planos profissionais tendo em vista a interface entre a carreira e os processos de saúde/doença (Guadassi et al., 2015; Schwitzer et al., 2018; Ramos et al., 2018).

Ademais, esses resultados precisam ser analisados com cautela, considerando o tamanho amostral e o tempo de aplicação dos instrumentos (imediatamente após a intervenção), sendo que a probabilidade das diferenças observadas pode estar relacionada à especificidade da amostra. Em especial, dificuldades relativas ao tamanho amostral para estudos com universitários têm sido relatado em outros estudos nacionais (Barbosa, Oliveira, Melo-Silva, \& Taveira, 2018). O GI e GC não foram equivalentes em termos de escores das variáveis, tendo o GI médias inferiores ao GC na maior parte das variáveis. Além disso, os participantes do GC não estavam buscando atendimento para as questões de carreira, diferente do GI, o que pode ser evidenciado pelas médias dos dois grupos. Talvez, por não estarem buscando atendimento (sendo oferecido após as respostas aos instrumentos nos diferentes tempos de coleta de dados), houve pouca adesão dos participantes do GC na resposta do questionário tem T2. Dessa forma, somente será possível afirmar, com segurança, que as mudanças no GI podem ser provavelmente extrapoladas para outras amostras quando aumentar a amostra tiver grupos equivalentes e realizar avaliação follow up, a fim de identificar a estabilidade das mudanças.

Apesar dessas limitações, este estudo apresenta direcionamentos importantes ao desenvolver uma intervenção baseada em um modelo teórico sequencial, teoricamente fundamentado na literatura e avaliado por um instrumento construído advindo do mesmo modelo teórico. Assim, sugere-se, para estudos futuros, que a avaliação de intervenções com foco no desenvolvimento de carreira de universitários seja realizada por instrumentos construídos a partir do modelo teórico que subsidiaram tais intervenções. A construção de programas voltados para esse público é importante considerando as demandas evidenciadas nesse contexto e que impactam, além do estabelecimento de metas, no desempenho acadêmico e na saúde mental deles (Ambiel et al., 2017; In, 2016; Marques, Gallager, \& Lopez, 2017; Guadassi et al., 2015). Ao mesmo tempo, seria interessante compreender os efeitos dessa intervenção em outras amostras, como em adolescentes e indivíduos em transição de carreira, 
a fim de auxiliá-los a construir metas e um planejamento de carreira condizente com seus interesses e com o mercado de trabalho.

\section{Referências}

Alves, C. F. (2018). Desenvolvimento e planejamento de carreira em universitários: Avaliação e intervenção baseadas no modelo Career Flow (Tese de doutorado). Universidade Federal do Rio Grande do Sul. Programa de Pós-Graduação em Psicologia. Porto Alegre.

Ambiel, R. A. M., Barros, L. O., Pereira, E. C., Tofoli, L., \& Bacan, A. (2017). Avaliação de processos de orientação profissional e de carreira: problemas e possibilidades. Avaliação Psicológica, 16(2), 128-136. doi: 10.15689/AP.2017.1602.02

Amundson, N., Goddard, T., Yoon, H. J., \& Niles, S. (2018). Hope-centred interventions with unemployed clients. Canadian Journal of Career Development, 17(2), 87-98. Recuperado de http://cjcdonline.ca/download/ hope-centred-interventions-unemployed-clients /

Audibert, A., \& Teixeira, M. A. P. (2015). Escala de adaptabilidade de carreira: Evidências de validade em universitários brasileiros. Revista Brasileira de Orientação Profissional, 16(1), 83-93. Recuperado de http:// pepsic.bvsalud.org/scielo.php?script=sci_arttext\& pid=S1679-33902015000100009

Bandura, A. (2001). Social cognitive theory: An agentic perspective. Annual Reviews Psychology, 52, 1-26. doi: 10.1146/annurev.psych.52.1.1

Barbosa, M. M. F., Oliveira, M. C., Melo-Silva, L. L., \& Taveira, M. C. (2018). Delineamento e avaliação de um programa de adaptação acadêmica no ensino superior. Revista Brasileira de Orientação Profissional, 19(1), 61-74. doi: 1026707/1984-7270/2019v19n1p61

Bastianello, M., Pacico, J., \& Hutz, C. (2014). Optimism, self-esteem and personality: Adaptation and validation of the brazilian version of the revised life orientation test (LOT-R). Psico-USF, 19(3), 523531. doi: 10.1590/1413-827120140190030

Brasil. (2016). Resolução no 510 de 07 de abril de 2016. Brasília: Conselho Nacional de Saúde.

Clarke, A., Amundson, N., Niles, S., \& Yoon, H. J. (2018). Action-oriented hope: An agent of change for internationally educated professionals.
Journal of Employment Counseling, 55(4), 155-165. doi: 10.1002/joec.12095

Cohen, J. (1977). Statistical power analysis for behavioral sciences. New York: Academic Press.

Creswell, J. W. W. (2010). Projeto de pesquisa: Métodos qualitativo, quantitativo e misto. 2 nd. ed. Porto Alegre: Bookman.

Davidson, O., Feldman, D., \& Margalit, M. (2012). A focused intervention for 1 st-year college studentes: Promotin hope, sense of coherence, and self-efficacy. The journal of Psychology, 146(3), 333-352. doi: 10.1080/00223980.2011.634862

Feldman, D., \& Dreher, D. (2012). Can hope he changed in 90 minutes? Testing the efficacy of a single-session goal-pursuit intervention for college students. Journal of Happiness Study, 13(4), 745-759. doi: 10.1007/s10902-011-9292-4

Feldman, D., \& Kubota, M. (2015). Hope, self-efficacy, optimism, and academic achievement: Distinguishing constructs and levels of specificity in prediction college grade-point average. Learning and Individual Differences, 37(1), 210-216. doi: 10.1016/j. lindif.2014.11.022

Feldman, D., Davidson, O., \& Margalit, M. (2015). Personal resources, hope, and achievement among college students: The conservation of resources perspective. Journal of Happiness Study, 16(3), 543560. doi: 10.1007/s10902-014-9508-5

Fouad, N., Ghosh, A., Chang, W., Figueiredo, C., \& Bachhuber, T. (2016). Career Exploration among College Students. Journal of College Student Development, 57(4), 460-464. doi: 10.1353/csd.2016.0047

Ginevra, M. C., Pallini, S., Vecchio, G. M., Nota, L., \& Soresi, S. (2016). Future orientation and attitudes mediate career adaptability and decidedness. Journal of Vocational Behavior 95-96(1), 102-110. doi: 10.1016/j.jvb.2016.08.003

Guadassi, R., Waser, A., \& Gati, I. (2015). Gender differences in the association of depression with career indecisiveness, career-decision status, and career-preference crystallization. Journal of Counseling Psychology, 62(4), 632-641. doi: 10.1037/ cou0000113

Hall (1996). Protean Careers of the 21st Century. Academy of Management Executive, 10(4), 6-16. Recuperado de https://www.jstor.org/stable/4165349 
In, H. (2016). Acculturation and hope as predictors of career decision self-efficacy among korean international undergraduate students. Journal of Career Development, 43(6), 526-540. doi: $10.1177 / 0894845316633784$

Koen, J., Klehe, U., \& Vianen (2012). Training career adaptability to facilitate a successful school-towork transition. Journal of Vocational Behavior, 81(3), 395-408. doi: 10.1016/j.jvb.2012.10.003

Lima-Dias, M., \& Soares, D. H. (2012). Planejamento de carreira: Uma orientação para universitários. Psicologia e Argumento, 30(68), 53-61. doi: 10.7213/ psicol.argum. 5884

Lovbond, P. F. (1998). Long term stability of depression, anxiety and stress syndromes. Journal of Abnormal Psychology, 107(3), 520-526. doi: 10.1037/0021-843X.107.3.520

Lovbond, P., \& Lovbond, S. (1995). The structure of negative emotional states: Comparison of the depression anxiety stress scales (DASS) with the Beck Depression and Anxiety Inventories. Behaviour Research and Therapy, 33(3), 335-343. doi: 10.1016/0005-7967(94)00075-u

Machado, W. L. (2013). A saúde mental como um estado completo: Instrumentos de avaliação e sua relação com qualidade de vida, personalidade e variáveis biossociodemográficas (Tese de doutorado). Universidade Federal do Rio Grande do Sul. Programa de Pós-Graduação em Psicologia do Desenvolvimento. Porto Alegre.

Marques, S. C., Gallagher, M. W., \& Lopez, S. J. (2017). Hope and Academic-Related Outcomes: A Meta-Analysis. School Mental Health 9(3), 250-262. doi: 10.1007/s12310-017-9212-9

Niles, S. G., Norman, A., \& Yoon, H. J. (2019). Hopeaction theory: Creating and sustaining hope in career development. In N. Arthur, R. Neault, \& M. McMahon (Eds.), Career theories and models at work: Ideas for practice (pp. 283-294). Toronto, ON: CERIC.

Niles, S. G., Amundson, N. E., \& Neault, R. A. (2010a). Career flow: A hope-centered approach to career development. Boston: Pearson Education.

Niles, S., In, H., \& Amundson, N. (2014). Using an action oriented hope-centered model of career development. Journal of Asia Pacific Counseling, 4(1), 1-13. doi: 10.18401/2014.4.1.1
Niles, S., Yoon, H., Balin, E., \& Amundson, N. (2010b). Using a hope-centered model of career development in challenging times. Turkish Psychological Counseling and Guidance Journal, 4(34), 101-108. Recuperado de https://dergipark.org.tr/en/download/ article-file/200146

Nota, L., Santilli, S., \& Soresi (2016). A Life-Design-Based Online Career Intervention for Early Adolescents: Description and Initial Analysis. The Career Development Quarterly, 64(1), 4-19. doi: 10.1002/cdq.12037

Obi, O. (2015). Constructionist career counseling of undergraduate students: An experimental evaluation. Journal of Vocational Behavior, 88(1), 215-219. doi: 10.1016/j.jvb.2015.03.009

Pacico, J., Bastianello, M., Zanon, C., \& Hutz, C. (2013). Adaptation and validation of the Dispositional Hope Scale for adolescentes. Psicologia: Reflexão e Crítica, 26(3), 488-492. doi: 10.1590/ S0102-79722013000300008

Park, I., Rie, J., Kim, H., \& Park, J. (2018). Effects of a Future Time Perspective - Based Career Intervention on Career Decisions. Journal of Career Development, 1(1), 1-15. doi: 10.1177/0894845318781043

Ramos, F. P., Andrade, A. L., Jardim, A. P., Ramalhete, J. L., Pirola, G. P., \& Egert, C. (2018). Intervenções psicológicas com universitários em serviços de apoio ao estudante. Revista Brasileira de Orientação Profissional, 19(2), 221-232. doi: 1026707/1984-7270/2019v19n2p221

Santilli, S., Marcionetti, J., Rochat, S., Rossier, J., \& Nota, L. (2017). Career adaptability, hope, optimism, and life satisfaction in italian and swiss adolescents. Journal of Career Development, 44(1) 62-76. doi: $10.1177 / 0894845316633793$

Santilli, S., Nota, L., \& Hartung, P. (2019). Efficacy of a group career construction intervention with early adolescent youth. Journal of Vocational Behavior, 111(1), 49-58 doi: 10.1016/j.jvb.2018.06.007

Santos, K., Araújo, T., \& Oliveira, N. (2009). Estrutura fatorial e consistência interna do Self-Reporting Questionnaire (SRQ-20) em população urbana. Cadernos de Saúde Pública, 25(1), 214-222. doi: 10.1590/S0102-311X2009000100023

Savickas, M. L. (1997). Career adaptability: An integrative construct for life-span, life-space theory. Career 
Development Quarterly, 45(3), 247-259. doi: 10.1002/ j.2161-0045.1997.tb00469.x

Savickas, M. (2013). Career construction theory and practice. Em R. W. Lent \& S. D. Brown (Eds.), Career development and counseling: Putting theory and research to work (pp. 147-183). Hoboken, New Jersey: John Wiley and sons.

Savickas, M., Nota, L., Rossier, J., Dauwalder, J., Duarte, M., Guichard, J., Soresi, S., ... \& Vianen, A. (2009). Life designing: A paradigm for career construction in the 21 st century. Journal of Vocational Behavior 75(3), 239-250. doi:10.1016/j.jvb.2009.04.004

Savickas, M. L., \& Porfeli, E. J. (2012). The Career Adapt-Abilities Scale: Construction, reliability, and measurement equivalence across 13 countries. Journal of Vocational Behavior, 80(3), 661-673. doi:10.1016/j.jvb.2012.01.011

Scheier, M. F., Carver, C. S., \& Bridges, M. W. (1994). Distinguishing optimism from neuroticism (and trait anxiety, self- mastery, and self esteem): A reevaluation of the Life Orientation Test. Journal of Personality and Social Psychology, 67(6), 1063-1078. doi: 10.1037//0022-3514.67.6.1063

Schwitzer, A. M., Moss, C. B., Pribesh, S. L., St. John, D. J., Burnett, D. D., Thompson, L. H., \& Foss, J. J. (2018). Students with mental health needs: College counseling experiences and academic success. Journal of College Student Development, 59(1), 3-20. doi: $10.1353 /$ csd.2018.0001

Snyder, C. (2002). Hope theory: Rainbows in the mind. Psychological Inquiry, 13(4) 249-275. doi: 10.1207/ S15327965PLI1304_01

Snyder, C. R., Harris, C., Anderson, J. R., Holleran, S. A., Irving, L. M., Sigmon, S. T., ... Harney, P.
(1991). The will and the ways: Development and validation of an individual-differences measure of hope. Journal of Personality and Social Psychology, 6(4), 570-585. doi: 10.1037//0022-3514.60.4.570

Spurk, D., Kauffeld, S., Barthauer, L., \& Heinemann, N. (2015). Fostering networking behavior, career planning and optimism, and subjective career success: An intervention study. Journal of Vocational Behavior, 87(1), 134-144. doi:10.1016/j.jvb.2014.12.007

Talib, J. A., Salleh, A., Amat, S., Ghavifekr, S., \& Ariff, A. M. (2015). Effect of career education module on career development of community college students. International Journal for Educational and Vocational Guidance, 15(1), 37-55. doi: 10.1007/ s10775-014-9279-x

Thompson, M., Her, P., \& Nitzarim, R. (2014). Personal and contextual variables related to work hope among undergraduate students from underrepresented backgrounds. Journal of Career Assessment, 22(4), 595-609. doi:10.1177/1069072713514814

Whiston, S. C., Li, Y., Mitts, N., \& Wright, L. (2017). Effectiveness of career choice interventions: A meta-analytic replication and extension. Journal of Vocational Behavior, 100(1), 175-184. doi: 10.1016/j. jvb.2017.03.010

Yoon, H. J., Bailey, N., Amundson, N. E., \& Niles, S. G. (2019). The effect of a career development programme based on the Hope-Action Theory: Hope to Work for refugees in British Columbia. British Journal of Guidance and Counselling, 47(1), 6-19. doi: 10.1080/03069885.2018.1544827

Recebido em: 04/12/2018

Reformulado em: 07/11/2019

Aprovado em: 02/01/2020 
Nota dos autores:

Financiamento: Coordenação de Aperfeiçoamento de Pessoal de Nível Superior (Capes) através de Bolsas de Doutorado para a primeira autor.

Sobre os autores:

Cássia Ferrazza Alves é Mestre e Doutora em Psicologia pelo Programa de Pós-Graduação em Psicologia/UFRGS, Professora de Psicologia da FSG Centro Universitário.

ORCID: https://orcid.org/0000-0003-0022-6467

E-mail: cassiaferrazza@gmail.com

Marco Antônio P. Teixeira é Doutor em Psicologia/UFRGS, Professor no Instituto de Psicologia/UFRGS e do Programa de Pós-Graduação em Psicologia/UFRGS.

ORCID: http://orcid.org/0000-0001-7981-9788

E-mail: mapteixeira.psi@gmail.com

Contato com os autores:

Cássia Ferrazza Alves

Rua Alfredo Chaves, 1208/sala 1411, Centro

Caxias do Sul-RS, Brasil

CEP: 95020-460 